\title{
The psychophysics of a human brain subjective activity
}

\author{
Evgenie A. Yumatov \\ Department of Normal Physiology, 1st Moscow State Medical University after Named of Sechenov, Moscow, Russia \\ Email: eayumatov@mail.ru
}

Received 11 February 2013; revised 13 March 2013; accepted 7 April 2013

Copyright (C) 2013 Evgenie A. Yumatov. This is an open access article distributed under the Creative Commons Attribution License, which permits unrestricted use, distribution, and reproduction in any medium, provided the original work is properly cited.

\begin{abstract}
Recently we have established the existence of a "psychogenic field", which are most likely reflecting on the human brain subjective state. The goal of this study is to further explore the human subjective state which can be registered remotely and objectively in the context of human subjective state. The analysis of the remote influence of a human subjective state on the blood physical and chemical parameters appeared to be main driving horse. The concept of specific physical phenomena in the brain does not exist in the abiocoen. Future exploring pattern of the relationships between the neurophysiologic and subjective processes in the systemic organization of "goal-seeking" behavior suggests that the paradigm presuming the existence of physical phenomena as a unique interrelationship for the active human brain, and their role in the origin of a subjective state.
\end{abstract}

Keywords: Subjective State; Brain; A Psychogenic Field; Paradigm of a Subjective State Origin

\section{INTRODUCTION}

The human brain is a unique organization in the living nature. The brain is able to self-perception, self-sensation, self-aesthesia, in fact the brain can be in subjective, spiritual state, meaning "subjective" as personal, spiritual, individual, i.e. the attitude to itself, to something or to somebody [1-4].

The subjective brain state reflects the perception of the life in all of its variety. The various forms of subjective state can be observed in living beings such as: sensations, feelings, emotions, consciousness; and can also create specific language, abstract and heuristic thinking as well as self-perception.

Every human experiences his/her own personal subjective state, which is very important for him/her. We feel the existence of similar subjective state in other people by the presence of our own sensations. The perception of life and the world around us are done through subjective sensations that vary greatly in individuals and depend upon the development, education, upbringing, culture, mentality, living standards, motivations, interests, health status, etc. The closer his/her own subjective state to a respective human is, the further understanding of its nature he/she will get.

If the subjective really exists, the subjective will be objective, though it takes place merely in the living organization of matter.

K. Popper [2008] wrote: "We live in the world of physical bodies and we ourselves are physical bodies. When I speak to you, I do not address your bodies, but your minds. Here arises the question of interaction between these two worlds, the world of physical states or processes and the world of mental states or processes. This question is a psychophysical problem [5]."

The brain activity is dual in nature. On one hand, there are neurophysiologic processes. On the other hand, there are subjective states, characterizing all facets of the world-view [6-12].

The neurophysiologic processes can be observed and registered by various methods and devices development based on laws of physics and chemistry. But we do not perceive the processes on the neurophysiologic level, for example, we do not feel the activity of a particular neuron.

The processes on the subjective level are perceived as self-sensations, which cannot be registered directly by physical and chemical methods based on the phenomena in the abiocoen.

The achievements in modern neurophysiology based on structural-morphological, electrophysiological, neurochemical, molecular, genetic research, on computer tomography, etc. do not allow identifying the spiritual functions of the brain. Whatever processes are registered: the electroencephalogram (EEG), neuronal activity, neurochemical and molecular reactions, etc., they do not show the internal experiences: thoughts, emotions, senses, etc. The computer tomography of a brain, mapping of 
genes expression in various brain structures, multichannel record of neuronal activity, multichannel electroencephalogram allow revealing only participation and interaction of various brain structures in the organization of behaviour, training, memory, emotions and thinking. However, these studies do not bring us to the closer understanding of origin of subjective states. Even there are no hypothetical, imaginary logical concepts in science that explain the origins of the subjective in neurophysiologic processes.

There is a huge gap between modern knowledge about brain neurophysiology and understanding of its mental functions. This is because the fact that the brain was studied with the morphological, physical and chemical methods based on the knowledge, phenomena and laws which discovered in the investigations of the abiocoen. The origin of a subjective brain state is beyond the field of hi-tech analytical research. This is their essential limitation. The objective research of a subjective state calls for different scientific methods and approaches.

A living organism and a brain in particular may be the place for physical phenomena and processes which are impossible in the abiocoen. This thesis is very important for understanding the essence of the subjective in the brain activity.

The brain activity has two facets: visible, characterized by registered neurophysiologic parameters, and hidden or spiritual, manifesting in human subjective perception of oneself and the world around him/her. No doubt, these to facets are interrelated. However, we cannot explain how the code of nerve impulses, the interacttion of various brain structures, the electrophysiological processes, the molecular transformations result in the self-perception of subjective state.

According to P. K. Anokhin [1969], "specific mechanisms of subjective consciousness generation cannot be described analytically, even though their exact information relation to the initial parameters of the objective world is beyond doubt" [7]. The subjective states of individuals reflect real psychophysiologic processes [12]. The question how the brain generates its inner spiritual world remains one of the greatest secrets of the Nature.

In our research, we try, without going into the details of particular subjective states manifestations: consciousness, emotions, etc. to find basic approaches to understanding the origin of the subjective in the brain activity. This research is based upon the methodological principle that we formulated "the subjective processes can be registered directly only by living structures" $[13,14]$. Using this principle, we carried out a variety of series researches in which for the first time established possibility of direct remote registration a subjective condition of the human with aid of the subjective state indicator $[15,16]$.

The findings suggest that a human subjective state can be registered objectively and remotely. It is important to note that a subjective state indicator shows his/her subjective attitude towards somebody or something.

The effect, which we found, characterizes specific abilities of individuals. It was found that about $10 \%$ of humans show their own subjective state in subjective state indicator. It is not surprising as everything is unique in the field of biology, from a unique DNA to diversity of individual forms of behavior and display of emotions.

We organized expert examination of our findings credibility with the participation of leading experts in physiology and physics: RAS Member A. S. Bugaev, RASM Member K. V. Sudakov, V. G. Zilov, Prof. O. V. Betsky, Prof. Y. I. Levin, Prof. V. V. Raevsky, Prof. Y. A. Khananashvili, whose peer-reviews are included into the monograph [15]. These reviews highlight the reliability and repeatability of our findings.

While the credibility of our findings do not raise doubts both in the experts and us, nevertheless, we wanted to have additional evidence on a direct objective registration in blind tests eliminating skeptical attitude towards our findings.

In our research, we hypothesized that the human subjective state may manifest itself in different forms. For registration of a human subjective state, we used of blood as a biological multicomponent substrate containing cell elements, protein-colloid and electrolyte solutions.

\section{MATERIALS, METHODS AND RESULTS}

\section{The Remote Influence of the Human Subjective State on the Physical and Chemical Properties of the Blood}

At these series of tests we try to find out if the remote direct influence of the investigators subjective on blood in particular on erythrocyte sedimentation rate (ERS) is possible.

We use blood samples taken from healthy individuals in a clinical setting. ERS was measured using standard Panchenko method. We compared ERS figures in three capillaries fixed in separate test tube racks.

In the first rack, we put a capillary with blood unexposed to any subjective influence (control). In the second rack, we put a capillary with blood approached by the investigator in a neutral subjective state holding the subjective state indicator in his hands (neutral). The pointers of the subjective state indicator remained in the initial position and were directed forward. The neutral control was necessary to exclude any possible outside influence of the investigator on ERS, e.g. associated with movement and approach of the investigator to the rack, with heat, electromagnetic and electrostatic influence of the 
investigator's clothes, etc.

The third rack with blood capillary was approached by the investigator in a certain subjective state and indicated by the crossed position of the pointers (subjective). The subjective state was provoked by the investigator with one of the tests described above $[15,16]$ : an expressed imagined emotional or cogitative condition.

The subjective state indicator pointers demonstrated the observer(s) what was the subjective state of the investigator when he/she approached to a blood filled capillary. While the investigator perceived his/her subjective state without indicators.

In the tests, the investigator approached the second rack five times in a row (neutral), and then 5 times in a row to the third rack (subjective) with a several second interval. In separate tests the number of approaches varied from one to ten. All three tests were performed at the same time with samples taken one person. All racks were put in vertical position simultaneously. The ERS was calculated in one hour.

We compared the ERS figures in three different capillaries: control, neutral and subjective. The findings were processed with standard mathematical methods to reveal regularity. The combined study was performed by E. Bykova and R. Jafarov.

The findings demonstrate reliable difference of ERS in control, neutral and subjective tests Figure 1. All subjective tests showed significant decrease of ERS in many times. The subjective state caused ESR decrease from 1.5 $\mathrm{mm}$ to $2.5 \mathrm{~mm}$. The higher was the baseline ESR figure in the control test of a particular individual, the bigger was the difference between ESR figures in a control and subjective tests. However, no reliable difference was found between the control and neutral tests that suggests

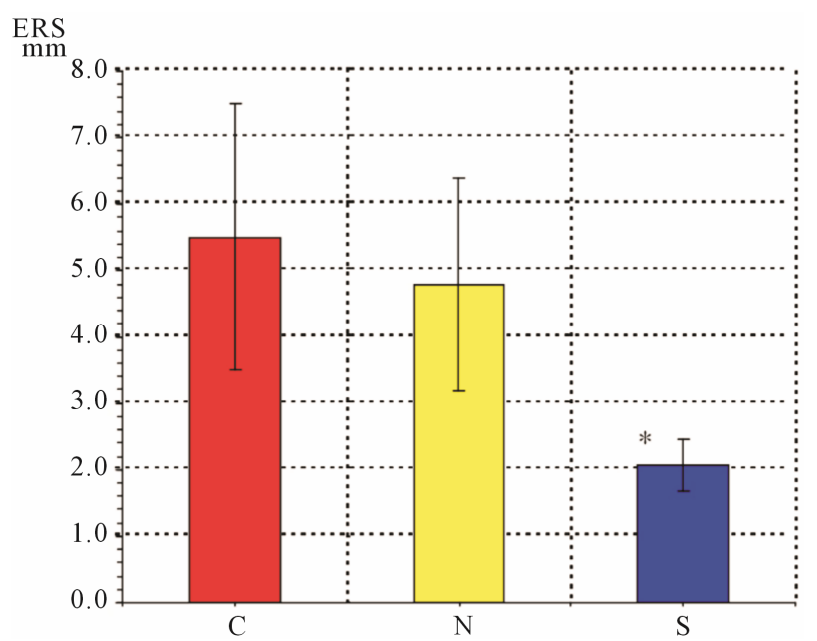

Figure 1. The change of erythrocytes sedimentation rate (ERS) in blood after the remote influence of the investigator subjective state. ERS mm. C-control, $\mathrm{N}$-neutral, $\mathrm{S}$ - subjective. Reliable difference ${ }^{*}-\mathrm{p} \leq 0.05$ between $\mathrm{C}, \mathrm{N}$ and $\mathrm{S}$. the blood was not affected by the neutral subjective state of an investigator.

Thereupon there was naturally a question, what is the distance on which a subjective state of the investigator influences on ERS of the blood? We have found that influence of a subjective state of the investigator on blood completely disappears at removal of the investigator on $0.8-1 \mathrm{~m}$ from the capillary with blood. Thus, the field remote effect depends on distance between the investigator and blood.

The findings have proved that there is a remote-field effect of a human subjective state on the blood.

\section{DISCUSSION}

\subsection{The Psychogenic Field of the Human Brain}

The remote impact of the subjective state can be originated by the field generated by the biological object oneself-by the human brain. We call this a "psychogenic field" since it reflects the psychic subjective state of a human $[15,16]$.

The presence of any field is proved by its impact on processes or physical bodies. The existence of a psychogenic field is revealed by the remote mechanical impact on the pointers indicating various subjective states of a human [17]. Another manifestation of the psychogenic field is its reliable remote impact on ESR. This field may have other properties that we do not know yet. The remote manifestation of the psychogenic field depends upon its strength and spatial orientation, which may account for individual differences.

These findings pose question about the origin of psychogenic field, about the substrate that generates it, and about biological infrastructures it affects? These questions necessitate further research.

The contemporary computer and information technologies allow modeling the central architectonics of behavior [18] in technical devices (robots, automatic control systems). However, we cannot obtain the presence of the subjective: 'motions, self-perception, etc. It means that the architecture of the function system organization is not enough to generate the subjective. The brain has something different that is impossible in the abiocoen.

Historically the biological science developed basing on the knowledge and achievements of abiocoen physics. As for the physical laws of living beings, these laws were overlooked by the traditional physics and biology. Researching into the subjective human brain states we for the first time approached the physical field processes that are possible only in living organisms. No technical means are capable to register spiritual state. Subjective states can be perceived only by living structures.

We see that the psychogenic field really exists; reflects the subjective state of a human, associates with the brain 
activity; causes forces affecting on the subjective state indicator; produces remote impact on the physical properties of blood; and therefore is of physical nature.

\subsection{The Neurophysiologic and Subjective Processes in the Functional System of Goal-Seeking Behavior}

In literature there are a lot of works on a problem of subjective human brain activities. Into this heading enter: philosophical articles; psychological in which external forms of mental activity are described; the clinical works considering violations of mental functions at various diseases; neurophysiological in which authors try to reveal correlation and codes of mental cognitive, sensory functions of a brain including the means of FMRT. However among them there are no articles on experimental studying of the nature (origin) of subjective brain activities. A number of researchers note that the subjecttive aspect of brain activities still remains to be one of secrets of the nature, and some of them consider that this problem is still unknown.

Recognizing the existence of a brain subjective state, psychophysiology still views the subjective as something separated and unrelated to the formation of goal-seeking behavior. T. Nagel [2001] wrote “...describing mental phenomena, "subjective reality" and coupling them with the neurophysiologic processes faces the "explanation gap" because the mental processes are physical and can not be referred to spatial and time coordinates. On the other hand, there are no grounds to say that the physical does not accompany the mental, but the question is how? The parallel description of the neurophysiologic processes and mental states caused by them (?) or accompanied by them (?) does not help answer the question how the behavior of a neuron network produces the subjective states, feelings, self-reflection and other phenomena of high order. Without the change of the fundamental concepts of the consciousness, the explanation gap can not be overcome [19].

This "gap" is mentioned by T.V. Chernigovskaya [2008]: “...neurosciences and philosophy of consciousness are much better without each other, one can say they do interfere with each other..." [20].

In other words, it is impossible to analyze the origin of the subjective basing on the contemporary neurophysiologic methods, and all speculations are counterproductive and are unrelated to the understanding of the nature of the subjective.

Several researcher inspired by the achievements in neurophysiology, molecular biology, nanotechnologies, predict the possibility of deciphering of "thinking codes", consciousness and image visualization of neuron pulse activity $[11,21]$. In fact, it is possible in some cases to see certain correlation between the activity of certain brain structures or neurons and the content side of thinking, speech, etc. This however does not mean that in these processes, one can understand the subjective.

Among the billions of brain neurons, one can always find neurons whose activity will correlate with a particular function of the body. At the same time, the information encoding may differ between individuals and social groups. Each neuron registered in the brain is individual and different from the others. The investigator will never find this neuron in this or any other individual.

S. Hameroff [2007] hypothetically connects emergence of subjective human brain activities with structure and function of neurons microtubules. The author suggests that subjective state of a brain arises at quantum level of its organization, and the brain is the quantum computer. According to the author brain processes relevant to consciousness extend downward within neurons to the level of cytoskeletal microtubules. An explanation for conscious experience requires (in addition to neuroscience and psychology) a modern form of pan-protopsychism in which proto-conscious quails are embedded in the basic level of reality, as described by modern physics [22].

However these theoretical views aren't supported with pilot studies and the evidence of their communication with subjective brain activities isn't produced. They don't allow to understand, how the brain, unlike computers and other lifeless systems, creates the internal subjective state, i.e. itself feels?

N.P. Bekhtereva [1990] wrote: “... it is doubtful that the full code of the mental processes will be deciphered only by analyzing the pulse activity of neurons and neuron population. The solution of the task lies not only in the sphere of the physiology and biochemistry of a living object, but also in the finest branch of biochemistry: the biology of molecular processes. It is very important to keep reasonable attitude to the material basis of phenomena and to conduct goal-seeking and deeper search towards its deciphering. At the same time, one should try to imagine what the ideal is without putting it into "reinforced concrete" bed of materialism? It is not worth sticking to the primitive materialism which results biologists worked in a corridor limited by invisible barbed wire" [23].

Analyzing the problem of the origin of the subjective A. N. Leontyev [1975] supposed that "no direct correlation of psychic and brain physiologic processes will not solve the problem" [24].

There are two components in the thought: its subjecttive nature and its specific content. When researchers write on the possibility to register a subjective state in the neurophysiologic processes, they forget what the subjective is and take the neurophysiologic processes as a real manifestation of the subjective in the brain activity. This means that they do not see any difference between the subjective and neurophysiologic processes and in fact 
identify them.

The theory of functional systems developed by P. K. Anokhin [18] and presented in the works of K. V. Sudakov [25] and many other researchers points at the main mechanisms in the brain activity, which may be associated with origin of emotions and thinking. However the central architecture of a behavioral act reflects only neurophysiologic component and fails to represent the organization of the subjective processes.

The contemporary concept of the system organization of goal-seeking behavior lacks the separate description of processes taking place on the neurophysiologic and subjective levels. The subjective is left "off-camera" and it is only presumed existing.

It is quite understandable that this situation is because there was no possibility to register objectively the manifestation of the subjective state in the brain.

In the system organization of a goal-seeking behavior, we split the brain functions taking place in the neurophysiologic and subjective levels and presented the in- teraction of the neurophysiologic and subjective processes in Figure 2.

All known neurophysiologic phenomena registered in the brain can be associated with a various main mechanisms of functional system of goal-seeking behavior [18]. In addition to that, there are processes in the functional system of goal-seeking behavior, which take place only on the subjective level and without which the brain activity is impossible.

The functional system of goal-seeking behavior has two interrelated levels of brain organization: neurophysiologic and subjective.

The system processes take place both in the neurophysiologic and subjective spheres of brain activity.

All processes in the brain start at the neurophysiologic level and then develop on the subjective level, which is a kind of a "shadow" of the neurophysiologic processes. A. M. Ivanitsky [10] showed that a sensor stimulus produces a neurophysiologic response followed by a subjective sensation.

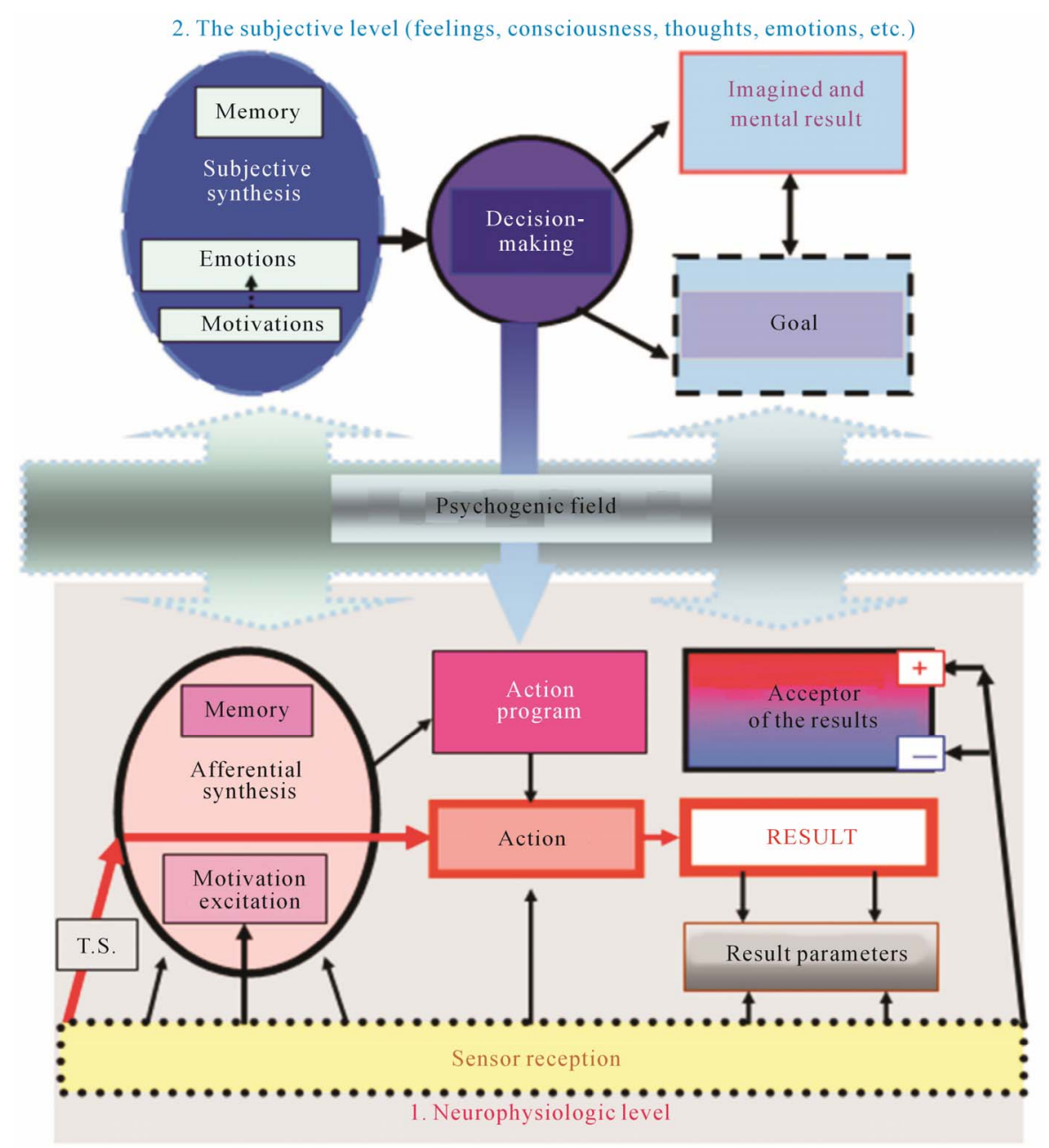

Figure 2. The hypothetical schematic drawing of the functional system in the context of the "goal-seeking" behavior: interaction of neurophysiologic (1) and subjective (2) processes in the brain activity. Abbreviations used in figure: T.S.- trigger stimulus. 
At the first stage, the afferential synthesis goes on the neurophysiologic level, and then the process of analysis and interpretation continues on the subjective level"subjective synthesis", including the sensation of attracttion (motivation), situational assessment, memorization and recall. The process is finished by decision-making and goal-setting made on the subjective level.

The further development of the processes in the brain may follow two ways.

In one case, the subjective synthesis finishes with the acceptation of an imagined mental result in the framework of subjective mental functional system the existence of which was highlighted by K.V. Sudakov [25].

In the other case, the decision about the goal-seeking behavior and goal-setting returns the processes from the subjective sphere to neurophysiologic level to formulate the program of result-oriented behavior and action results acceptors, in which the parameters of the future result is prognoses in accordance with the set goal.

Then, in the neurophysiologic level framework, everything develops according to the known scheme: an action is formed aimed at the achievement of a necessary result, parameters are registered, and through the reversed afferentation channels, these parameters go to the acceptor to be compared with parameters of the prognoses result.

If the prognoses and achieved results match, the process is stopped and a new stage of goal-seeking behavior without the participation of subjective level of the functional system.

If the prognoses and achieved results match, the result acceptor initiates a "mismatch" or "surprise" reaction. The process goes to the subjective level where negative or positive emotion is generated depending upon the failure of no-failure to achieve the goal.

The positive emotion produces satisfaction and fixation finishing a specific behavioral act. The negative emotion mobilizes the process of subjective synthesis to search another more appropriate decision that will allow achieving the goal.

The emotions are the means of memory fixation of the whole bunch of factors promoting or hindering achieving the goal. At the neurophysiologic level, new emotions are transformed into emotional reactions of the body. The behavior is oriented along the common vector from a negative reaction to a positive one.

The emotional reactions are formed in the neurophysiologic part of the functional system, while the emotions and thoughts are produced in the subjective part. A thought reflects the informational content of the prognosis and necessary result and emotions reflect the probability characteristic of possibility and feasibility to achieve the goal.

At the neurophysiologic level, the tailored reflex reac- tion and automatic behavioral acts are performed due to preoperational integration, which is formed earlier [18]. In these cases, the behavioral choice takes place without the participation of consciousness, and the interpretation may follow the action if the prognoses result was not achieved, or the interpretation may be omitted if a certain result is achieved.

The neurophysiologic level lies in the basis of perception of the environment and the internal state of the body, of organization of various behavioral forms and the body vital functions regulation.

The freedom of will, behavioral choices, imagined result and assessment of goal achievement tale place at the subjective conscious level.

We think that the consciousness is the ability of the brain to perceive its own state and the environment and take decisions, anticipate and assess the results of the actions. The consciousness includes emotions, feelings and thoughts that eventually determine the freedom of choice, decision-making and the result assessment.

The children first develop sensual, creative consciousness. In their subjective consciousness the children to the least extent observe themselves, reflecting over their thoughts and analyze their actions. The process of self-consciousness in children takes place in a "childadult" system where the adult is an intelligent volitional force directing the thoughts and actions of the child. As the child grows older, this connection weakens and the child gradually acquires the function of self-consciousness.

Decision-making and behavioral choices are always determined. The feeling of unrestricted freedom and the independence of choice are illusion, i.e. self-perception in one's consciousness. If we observe all the possibilities of an individual's actions, we see in fact that the individual had several possibilities and chose one. If we could see the processes in the individual's brain we would see that the choice was determined and the only possible basin on the combination and layout of all components of subjective and objective processes at a given moment of time.

It is not important for the individual what the freedom choice is: an illusion or really one's choice. Subjectively, he/she perceives this process as his/her decision. The freedom of choice is influenced by education, schooling, culture, etc., which form the perception of moral, laws, crimes, etc. That is why an individual bears the personal responsibility.

The subjective spiritual state of the brain results from the interaction and interrelation of neurophysiologic processes and brain-specific fields. Subjective and objective processes in the brain are closely interrelated and this relation is dual. The thought, consciousness and emotions manifest themselves not in a corpuscular matter 
but in its specific field form. This is a unique feature of the brain as a living organization of matter in the existing world.

The nature of a subjective ideal state is in the objective processes of the brain activity. The subjective is a phenomenon, function, state of brain, which, we think, emerges in the interaction of structural and molecular (neurophysiologic) and field processes in a living brain.

The electric physiology of excitable structures suggests that the electromagnetic field generated by a nerve cell affects the excitability and can cause excitation and conduction of a nerve pulse. We think that the fields generated by the brain produce reverse influence on the neurophysiologic mechanisms of the brain. Following the physical analogy we can call the reverse influence of the field on the structural and molecular processes in the brain the "brain self-induction".

The origin of a subjective brain state is in the fundamental properties of a living brain, which is a specific type of matter and has its own physical laws and specific brain fields. We think that one should not identify the notions of the information and the subjective. The information is present both in inanimate and animate objects. The notion of information does not define the nature of and is not an analog of the subjective. The information should not be viewed as an ideal substance above the real processes. The information is a link function among the elements of the system [15].

The central architectonics of the goal-seeking behavior functional system consists of two interrelated and united subsystems: a structural and neurophysiologic one and a subjective one. The structural and neurophysiologic component of the functional system cannot perform an effective activity without the participation of the subjecttive sphere, and the subjective subsystem is formed based on neurophysiologic processes.

The research into the neurologic, mental diseases is often limited to the objective neurophysiologic processes thus the researchers want to see them as the cause of the brain functional disorders. However, the origin of many brain diseases is in the subjective sphere of brain activity, which indirectly manifests themselves in the neurophysiologic processes. This may be the explanation of the unsolvable problems in the understanding of ethiology and pathogenesis of many psycho neurologic disorders.

The subjective state of a human has independent significance: it determines the recovery, treatment efficiency, disease outcome, and the successful psychotherapy is associated with the pact on the subjective sphere.

The Paradigm of the "Subjective": Basic Postulates.

The world-known expert in the field of scientific methodology T. Kuhn [1962] wrote: "The decision to decline a paradigm is simultaneously a decision to take another paradigm, and the sentence bringing us to this solution includes both comparison of both paradigms with nature and the comparison of the paradigms to each other" [26]. The science lacks any paradigm about the origin of a subjective state besides common view that a subjective state is in a way originated in the systemic brain mechanisms and neurophysiologic processes.

Since the organization of brain activity systemic and basing on our tests of human subjective state registration, we came to the basic postulates given below, which characterize interrelation of subjective and objective processes in a human brain.

- The subjective state of a brain manifests itself not in the corpuscular organization of a living structure but in a specific field form- - "psychogenic field".

- The subjective states can be perceived only by living structures.

- The biological fields produced by the body can directly affect the structural and functional processes in the body ("biological self induction").

- The subjective spiritual state of the brain results from interaction and interrelation of neurophysiologic processes and brain-specific biological fields.

- Psycho neurologic diseases can primarily occur in the subjective processes and secondarily manifest themselves in various structural and functional disorders.

- Living brain physics is a new field of science analyzing unique physical phenomena characteristic only to a living brain and non-existent in abiocoen.

\section{REFERENCES}

[1] Pavlov, I.P. (1951) The twenty years of objective study of animal higher nervous activity (behavior). In: Pavlov, I.P., Ed., Complete Compilation of Works, The Publishing House of the Academy of Sciences of the USSR, Leningrad (in Russian).

[2] Damasio, A. (2000) The feeling of what happens: Body and emotion the making of consciousness. Harcourt Brace, New York.

[3] Edelman, G. and Tononi, G. (2000) Consciousness. How matter becomes imagination. Penguin Books, London.

[4] Reber, A. and Reber, E. (2001) Dictionary of psychology. 3rd Edition, Penguin/Viking, London.

[5] Popper, K. (2008) Knowledge and psychophysical problem: In protection of interaction. Moscow. Transfer with English Zhuravlyov, I.V., Lietuvių Kalbos Institutas, Moscow (in Russian).

[6] Sperry, R.W. (1952) Neurology and the mind-brain problem. The Journal of American Science, 40, 291-312.

[7] Anokhin, P.K. (1969) The psychic for of the reality reflection. In: Pavlov, T., Ed., Lenin's Theory of Reflection, Science and Art, Sofia, Part 1, Chapter 3, 109, (in Russian).

[8] Bekhtereva, N.P., Budsen, P.V. and Gogolotsin, Y.L. (1977) Brain codes of psychic activity. Science, Lenin- 
$\operatorname{grad}$ (in Russian)

[9] Ivanitsky, A.M. (1999) The main secret of nature: How brain processes result in subjective experience. Psychological Journal, 20, 93-104 (in Russian).

[10] Ivanitsky, A.M. (2005) Consciousness and brain. Journal in the World of Science, 11, 1-8 (in Russian).

[11] Koch, C. (2005) Neurobiology of consciousness. MIT Press, Cambridge.

[12] Sudakov, K.V. (2010) System mechanisms of psychic activity. Korsakov Journal of Neurology and Psychiatry, 110, 4-14 (in Russian).

[13] Yumatov, E.A. (2010) Information and field effects of registration of the human brain subjective state. Clinical Informatics and Telemedicine, 6, 98-104 (in Russian).

[14] Yumatov, E.A. (2010) The direct registration of a human subjective state. Bulletin of new medical technologies, $\mathbf{4}$, 187-192 (in Russian).

[15] Yumatov, E.A. (2011) System psychophysiology of human subjective state. Sputnik + Moscow (in Russian).

[16] Yumatov, E.A. (2011) The physics of human brain subjective state. Consciousness and Physical Reality, 10, 39-50 (in Russian).

[17] Yumatov, E.A. (2012) About the nature subjective in activity of a brain of the person. Russian Medicobiological Bulletin, 2, 116-131 (in Russian).
[18] Anokhin, P.K. (1968) The biology and neurophysiology of conditional reflex. Medicine, Moscow (in Russian).

[19] Nagel, T. (2002) Think ability of impossible and problem of a spirit and a body. Philosophy Questions, 10, 92-107 (in Russian).

[20] Chernigovskaya, T.V. (2008) The human in a human: Consciousness and neuron network. In: The Problem of consciousness in Philosophy and Science, RAS Institute of Philosophy, Moscow, "Canon+" (in Russian), pp. 143163.

[21] Anokhin, K.V. (2010) Lecture on the brain codes, (in Russian). http://nature-wonder.livejournal.com/175914.html

[22] Hameroff, S. (2007) Consciousness, neurobiology and quantum mechanics: The case for a connection. In: Tuszynski, J., Ed., The Emerging Physics of Consciousness, Springer-Verlag, Berlin.

[23] Bekhtereva, N.P. (1990) Per aspera... life. Science about a brain of the human. Science, Leningrad (in Russian).

[24] Leontyev, A.N. (1975) Activity, consciousness, personality. Politizdat, Moscow (in Russian).

[25] Sudakov, K.V. (2010) On the theory of the unity of the material and ideal in the human activity. Journal of $\mathrm{Hu}$ man, 6, 5-16 (in Russian).

[26] Kuhn, T. (1962) The structure of scientific revolutions. University of Chicago Press, Chicago. 